\title{
Giant Aortic Aneurysm in Child with Cutis Laxa Syndrome: Unusual Presentation, New Surgical Technique
}

\author{
Mazen Shamsaldeen Faden, MD,${ }^{1}$ Nada Ahmed Noaman, MBBS,${ }^{1}$ Osman Osama Al-Radi, FRCSC, ${ }^{2}$ \\ Ahmed Abdelrahman Elassal, MD, ${ }^{2,3}$ Arwa Mohammed Al-ghamdi, MS, ${ }^{4}$ Ragab Shehata Debis, MD ${ }^{2,5}$ \\ ${ }^{1}$ Department of Anesthesia and Critical Care, King Abdul-Aziz University Hospital, Jeddah, Kingdom Saudi Arabia; \\ ${ }^{2}$ Department of Surgery, Cardiac Surgery Unit, King Abdul-Aziz University, Jeddah, Saudi Arabia; \\ ${ }^{3}$ Cardiothoracic Surgery Department, Zagazig University, Zagazig, Egypt; \\ ${ }^{4}$ Cardiac Surgery Unit, Co-ordination Unit, King Abdul-Aziz University, Jeddah, Saudi Arabia; \\ ${ }^{5}$ Cardiothoracic Surgery Department, Al-Azhar University, Cairo, Egypt
}

\section{ABSTRACT}

Ascending thoracic aortic aneurysms are rare in childhood and typically are seen in the setting of connective tissue defect syndromes. These aneurysms may lead to rupture, dissection, or valvular insufficiency, so root replacement is recommended. Here, we present a 17 -month-old girl who presented with fever, cough, and pericardial effusion. Initially, we suspected this could be a COVID-19 case, so a nasopharyngeal swap was performed. An ascending aorta aneurysm involving the aortic arch was confirmed by echo, and urgent ascending aorta and arch replacement were done by utilizing the descending aorta as a new arch. The final diagnosis came with cutis laxa syndrome. In similar cases, good outcomes can be achieved with accurate diagnosis and appropriate surgical management.

\section{INTRODUCTION}

Congenital ascending aortic aneurysm in children is a rare cardiovascular pathology and usually associated with connective tissue disorders [Tireli 2019]. Other causes include aortic wall abnormalities, aortic valve diseases or in association with other congenital cardiac defects [Alhabdan 2011]. There is a lack of established guidelines for diagnosis and treatment options for aortic aneurysms in neonate, infants, and children [Landis 2015]. In childhood, there are no definitive descriptions to guide the timing of surgery. Early surgery often is undertaken, given the emergence of significant aortic regurgitation or a rapid rate of growth $(>1 \mathrm{~cm}$ in a year) [Mehdi 2016].

Received Fune 20, 2021; received in revised form September 22,2021; accepted October 4, 2021

Correspondence: Ragab Shehata Debis, MD, Consultant Cardiac Surgery, King Abdul-Aziz University, Department of Surgery, Cardiac Surgery Unit, Jeddab Saudi Arabia, Telephone/Fax +966 12 6408222/13321, +966569806744 (e-mail: Openheart32@gmail.com).

\section{CASE REPORT}

We present the case of a 17 -month-old girl, whose mother and father are first cousins. She presented to our hospital with a history of fever and cough for 5 days, associated with decreased feeding and decreased activity. She had no history of contact with a sick patient and no history of significant weight loss. The first provisional diagnosis that came to mind was COVID-19 infection, so a nasopharyngeal swap was performed. On examination, the child was fully conscious and hemodynamically stable with congested neck veins and a muffled heart sound. The chest X-ray showed a huge pericardial shadow (Figure 1A), bedside echo showed a large circumferential pericardial effusion (Figure 2A). (Figure 1) (Figure 2) The initial plan was pericardiocentesis, which was done and
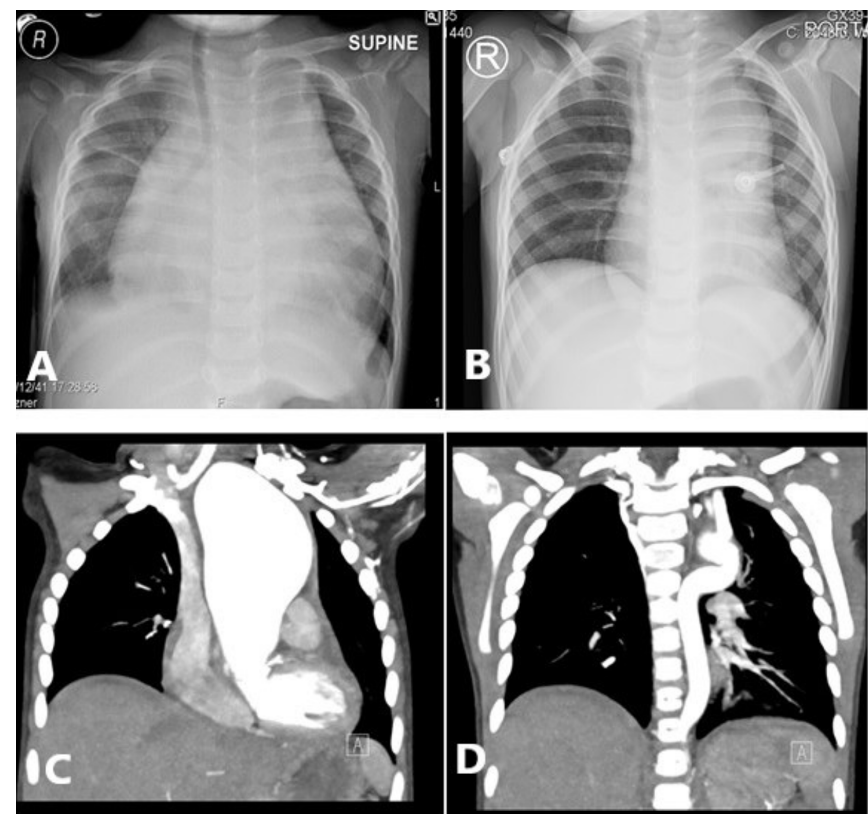

Figure 1. A) Chest X-ray with massive pleural effusion. B) Chest X-ray post effusion drainage wide mediastinum. C) CT of chest showing giant ascending aortic aneurysm. D) CT chest showing elongated tortuous descending aorta. 

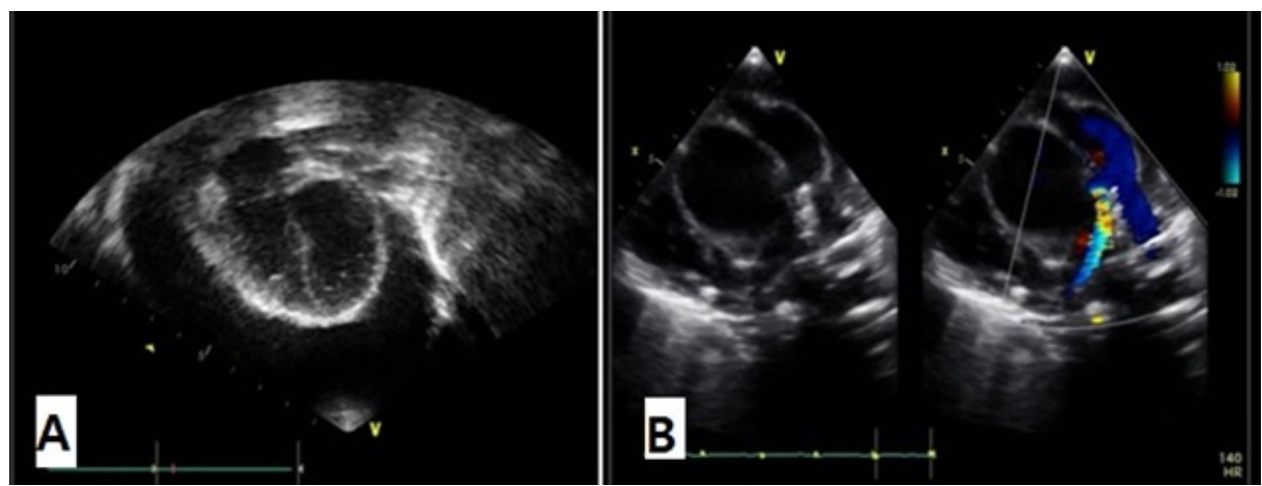

Figure 2. Echocardiography. A) large pericardial effusion. B) dilated ascending aorta compressing right pulmonary artery and left atrium.
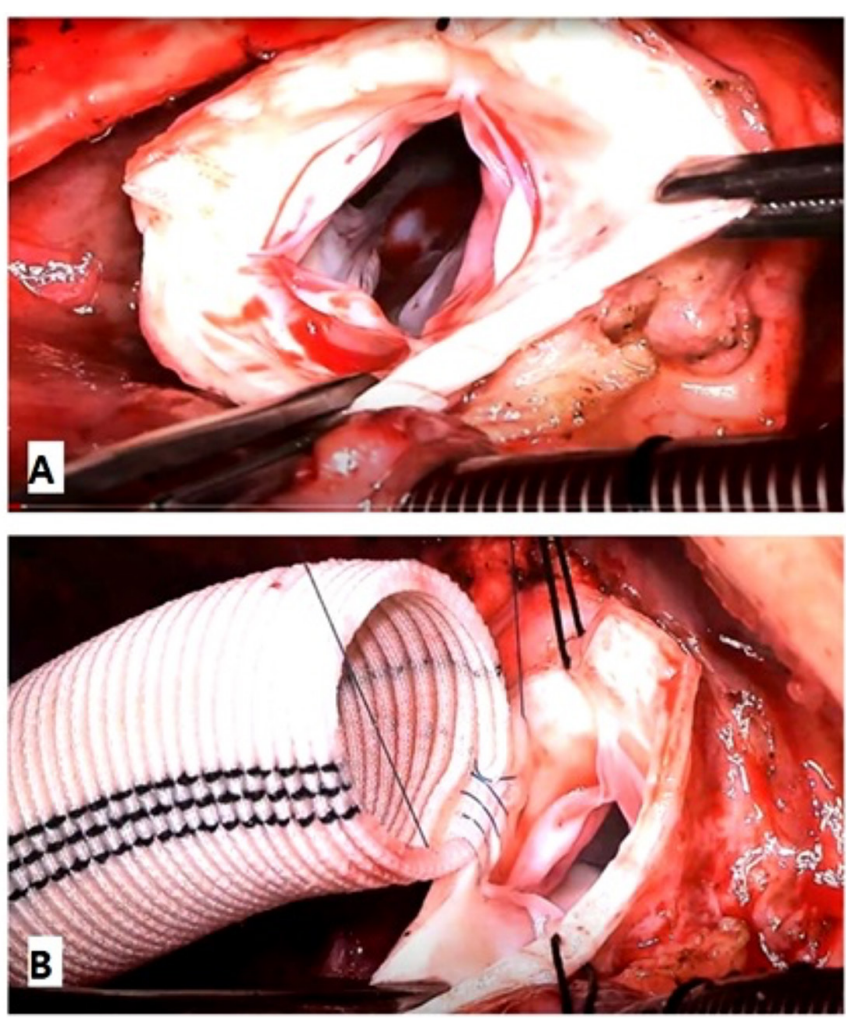

Figure 3. A) Normal trileaflet aortic valve. B) supracoronary anastomosis of tube graft.

revealed hemorrhagic effusion, pigtail inserted. Chest X-ray post drainage showed wide mediastinum (Figure 1B), cardiac computed tomography showed a dilated ascending aorta and aortic arch with a maximum diameter of $4.4 \mathrm{~cm}$, no associated anomalies, and elongated tortuous descending thoracic aorta (Figure 1D). Echocardiography showed a dilated ascending aorta and compressed right pulmonary artery (Figure $2 \mathrm{~B}$ ). The COVID-19 result was negative, and the patient was prepared for urgent surgery.

The child underwent replacement of the ascending aorta and aortic arch under deep hypothermic $\left(18^{\circ} \mathrm{C}\right)$ with cardiopulmonary bypass, del-Nido cold blood cardioplegia with antegrade cerebral perfusion through the innominate artery. Intraoperatively, the ascending aorta and aortic arch were confirmed to be extremely dilated, the aortic valve was trileaflet, and structurally normal (Figure 3A). (Figure 3) The aortic sinuses and annulus were not involved. Excision of the ascending aorta and aortic arch with separation of great vessels were done, descending aorta looks healthy, elongated and tortuous (Figure 4A), so it pulled up to make neo aortic arch, and the great vessels were anastomosed to the new arch separately. (Figure 4) A $16 \mathrm{~mm}$ haemshield Dacron graft (DuPont, Wilmington, DE) was anastomosed proximally at the supracoronary level (Figure 3B) and distally to the proximal neo aortic arch (Figure 4B and 4C). The patient successfully was weaned from bypass, had good hemostasis, and the chest was closed.

Histologically, the aortic wall showed fibro-myxoid degeneration, loss of smooth muscle cells, fibrinoid necrosis, and infiltration with chronic inflammatory cells. Genetic test revealed cutis laxa syndrome, and the patient's postoperative course was uneventful. She has discharged on postoperative day seven. Follow-up echocardiographic examination showed stable hemodynamics, excellent morphologic and functional relationships of the aortic valve, ascending aorta, and arch, and competent aortic valve, as well as excellent left ventricular function.

\section{DISCUSSION}

Aortic aneurysms are very rare in infancy and childhood. They usually are associated with connective tissue abnormalities affecting collagen synthesis, such as Ehlers-Danlos syndrome, Marfan syndrome, Loeys-Dietz syndrome, or various other infections and trauma. All of these lead to disturbances in blood flow, increased vessel wall stress, and decreased nutrient supply to the arterial wall which result in dilatation and aneurysm formation [Tireli 2019]. In our case, the genetic test came with Cutis Laxa (CL) syndrome, which is a rare group of multisystem disorders resulting from impaired elastic fiber assembly and homeostasis, and the known underlying gene defects affect different extracellular matrix proteins, intracellular trafficking, or cellular metabolism [Beyens 2021]. 

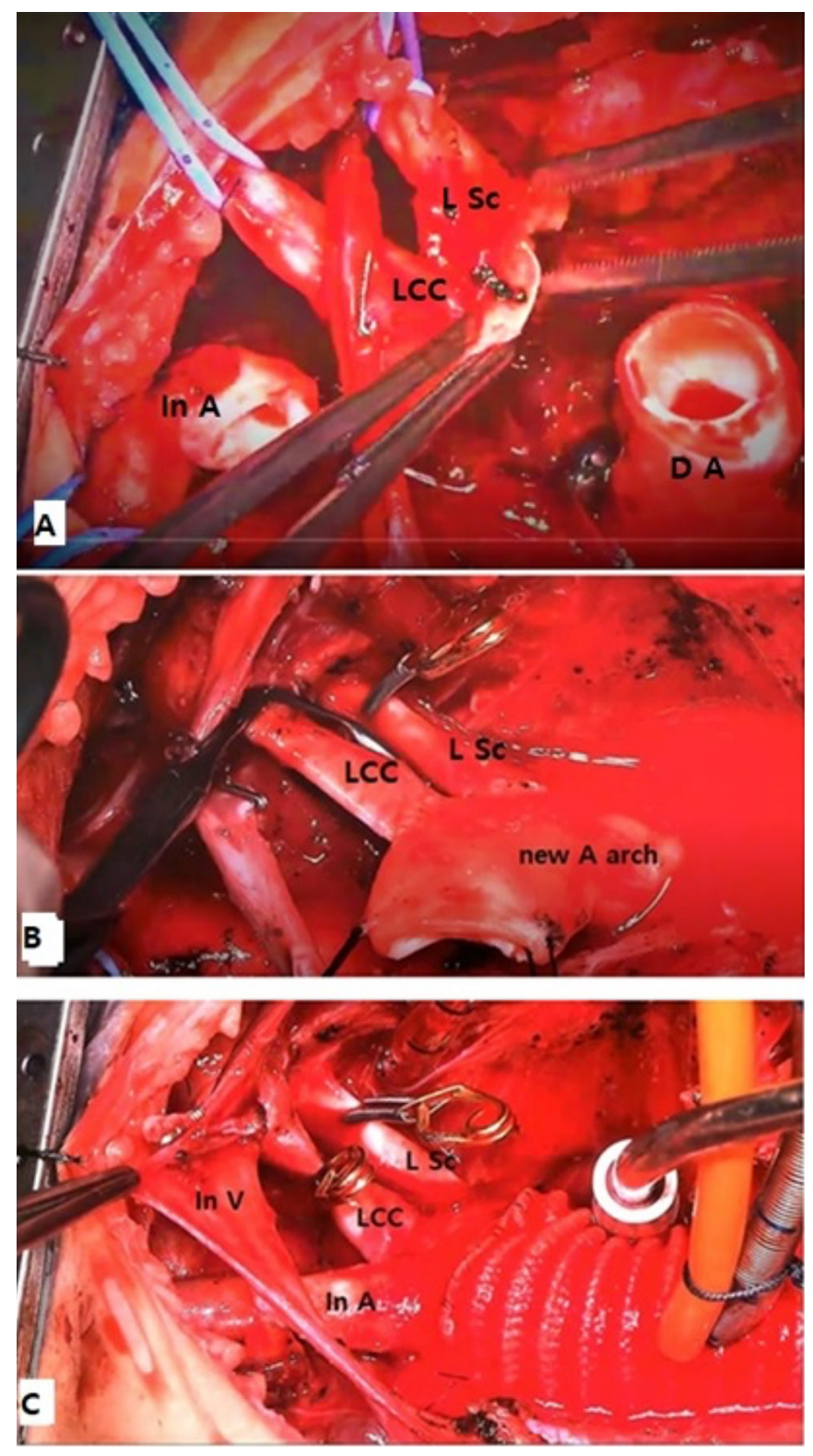

Figure 4. A) Excision of ascending aorta and aortic arch. B) reimplantation of left subclavian artery and left common carotid to new aortic arch. C) reimplantation of great vessels to new aortic arch and sewing proximal arch to tube graft. In A, innominate artery; D A, descending aorta; L Sc, left subclavian artery; LCC, left common carotid artery

The girl presented mainly with cough, fever, and pericardial effusion at 17 months of age. After the pericardial effusion was drained, cardiac CT was done and showed the aneurysm involved the ascending aorta and aortic arch with a maximum diameter of $4.4 \mathrm{~cm}$, which was far from index diameter for her age and weight. Due to high incidence of rupture, dissection, and the compression on surrounding structures, the patient was scheduled for urgent surgery. Although there is a lack of established guidelines for diagnosis, the treatment of aortic aneurysms in the childhood period, echo and cardiac CT remain the gold standard for diagnosis. Challenges for the management of aortic aneurysms in children include (1) failure to diagnose and institute treatment;(2) inability to exactly predict the aortic diameter at which dissection occurs in each disease; (3) unknown durability of valve-sparing root procedures; and (4) the unknown late vascular complications after pre-emptive surgery, especially in the more aggressive forms of Loeys-Dietz and Ehlers-Danlos syndromes. However, elective aortic root replacement remains the single most important intervention to improve life expectancy for young syndromic patients with aneurysms of the aortic root [Zanotti 2008]. The options for the replacement of aortic aneurysms in children are limited, with the most commonly used conduit being artificial graft. The main problem with the use of artificial blood vessels in children is that they may become relatively narrow compared with normal blood vessels as children mature and affect growth and development [Karthekeyan 2015]. In our case, we were lucky to have enough length of the descending aorta to make a new arch that gave us the chance to implant the great vessels to native tissue and avoid future narrowing and arch replacement in the future.

\section{REFERENCES}

Alhabdan MS, AlSehly AA. 2011. Ascending aortic aneurysm. Pediatr Cardiol 2011; 32:1266.

Beyens A, Boel A, Symoens S, Callewaert B. 2021. Cutis laxa: A comprehensive overview of clinical characteristics and pathophysiology. Clinical Genetics. 99:53-66.

Karthekeyan BR, Vakamudi M, Thangavel PS. 2015. Repair of idiopathic ascending aortic aneurysm in a 7-year-old child. Cardiology in the Young. 25, 154-157.

Landis BJ, Ware SM, James J, Shikany AR, Martin LJ, Hinton RB. 2015. Clinical stratification of pediatric patients with idiopathic thoracic aortic aneurysm. J Pediatr. 167:131-7.e1-5.

Mehdi G and Ghafar G. 2016. A Huge Aortic Aneurysm in a boy with Marfan Syndrome J Cardiol \& Cardiovasc Ther 1(4). JOCCT. MS.ID.555569.

Tireli E, Polat TB, Oztas DM, Mamur Y, Omeroglu RE, Ugurlucan M. 2019. Giant Aneurysm of the Ascending Aorta Requiring Emergency Repair in a Newborn Baby. Ann Thorac Surg. 107:e107-9.

Zanotti G, Vricella L, Cameron D. 2008. Thoracic Aortic Aneurysm Syndrome in Children. Pediatr Card Surg Ann. 11:11-21. 